\title{
Influence of index contrast in two dimensional photonic crystal lasers
}

Jørgensen, Mette Marie; Petersen, Sidsel Rübner; Christiansen, Mads Brøkner; Buss, Thomas; Smith, Cameron; Kristensen, Anders

Published in:

Applied Physics Letters

Link to article, DOI:

$10.1063 / 1.3443718$

Publication date:

2010

Document Version

Publisher's PDF, also known as Version of record

Link back to DTU Orbit

Citation $(A P A)$ :

Jørgensen, M. M., Petersen, S. R., Christiansen, M. B., Buss, T., Smith, C., \& Kristensen, A. (2010). Influence of index contrast in two dimensional photonic crystal lasers. Applied Physics Letters, 96(23), 231115.

https://doi.org/10.1063/1.3443718

\section{General rights}

Copyright and moral rights for the publications made accessible in the public portal are retained by the authors and/or other copyright owners and it is a condition of accessing publications that users recognise and abide by the legal requirements associated with these rights.

- Users may download and print one copy of any publication from the public portal for the purpose of private study or research.

- You may not further distribute the material or use it for any profit-making activity or commercial gain

- You may freely distribute the URL identifying the publication in the public portal

If you believe that this document breaches copyright please contact us providing details, and we will remove access to the work immediately and investigate your claim 


\title{
Influence of index contrast in two dimensional photonic crystal lasers
}

\author{
Mette Marie Jørgensen, Sidsel Rübner Petersen, Mads Brøkner Christiansen, a) \\ Thomas Buß, Cameron L. C. Smith, and Anders Kristensen \\ Department of Micro and Nanotechnology, DTU Nanotech, Technical University of Denmark, Building 345 \\ East, DK-2800 Kongens Lyngby, Denmark
}

(Received 22 April 2010; accepted 13 May 2010; published online 11 June 2010)

\begin{abstract}
The influence of index contrast variations for obtaining single-mode operation and low threshold in dye doped polymer two dimensional photonic crystal $(\mathrm{PhC})$ lasers is investigated. We consider lasers made from Pyrromethene 597 doped Ormocore imprinted with a rectangular lattice $\mathrm{PhC}$ having a cavity in the middle of the crystal structure. We demonstrate that the index contrast, $n_{\text {eff,high }} / n_{\text {eff,low }}$, is an essential parameter for achieving low threshold, and we identify a trade-off between low threshold and single-mode operation. (C) 2010 American Institute of Physics.

[doi:10.1063/1.3443718]
\end{abstract}

In the past few years polymer photonic crystal $(\mathrm{PhC})$ lasers have been rigorously studied, ${ }^{1-8}$ and $\mathrm{PhC}$ band edge lasing has been demonstrated in both organic and semiconductor material. Two dimensional (2D) polymer PhCs, used to create distributed feedback lasers, have in addition enabled low threshold operation and well-defined output beams. ${ }^{9,10}$ By adjusting the period, number of periods, and index contrast of the $\mathrm{PhC}$, it is also straightforward to control their operation mode. In particular, the index contrast is given by the ratio of the effective refractive indices $\left(n_{\text {eff }}\right)$ in the high and low $n_{\text {eff }}$ segments: $n_{\text {eff,high }} / n_{\text {eff,low }}$. We consider dye doped organic material, namely, the organic dye Pyrromethene 597 (PM597) (Refs. 11 and 12) mixed into the organic-inorganic hybrid material Ormocore. ${ }^{13}$ The 2D PhC is imprinted into a slab waveguide creating a periodicity of index variations, $n_{\text {eff,high }}$ and $n_{\text {eff,low }}$. The lasers are made by combined nanoimprint and photolithography $(\mathrm{CNP}),{ }^{14}$ enabling the PhC imprint and the definition of the laser area to be made in a single parallel processing step. In CNP the subwavelength features are physically deformed, while the larger structures are defined by UV exposure through the hybrid mask/mold. ${ }^{15} \mathrm{CNP}$ gives a potential for high throughput and low cost fabrication. We focus on PhCs with rectangular lattices and a quarter-wave phase shift line defect cavity located in the middle of the crystal, see Fig. 1. By introducing this cavity, single-mode operation can be assured, and the devices lase at the center frequency of the bandgap rather than at the band edges. We investigate how an increased index contrast leads to a lower threshold and identify a trade-off between optimal conditions for low threshold and single-mode operation. Furthermore, we observe that the loss of single-mode operation leads to reduced lifetimes. We show that dye doped polymer 2D PhC lasers with index contrast of 1.010 have the lowest threshold and a single-mode yield of $93 \%$. Therefore, the largest possible index contrast is not necessarily desirable but should be optimized.

The index contrast is varied by altering the imprint depth of the PhCs in seven steps $\{40,70,90,130,180,218,273\}$ $\mathrm{nm}$ corresponding to index contrasts in the range [1.004;

\footnotetext{
${ }^{\text {a) }}$ Author to whom correspondence should be addressed. Electronic mail: mads.christiansen@ nanotech.dtu.dk.
}

1.034]. The lasers are $100 \times 250 \mu \mathrm{m}^{2}$ large with imprinted PhCs having the lattice vectors $a=193 \mathrm{~nm}$ and $b=355 \mathrm{~nm}$ and hole size of $97 \times 178 \mathrm{~nm}^{2}$. The initial polymer film thickness of $423 \mathrm{~nm}$ is kept constant to maintain gain volume, and a cavity of $193 \mathrm{~nm}$ width is placed in the middle of the $\mathrm{PhC}$ structures.

The lasers are modeled numerically with the following two programs: Finite-difference time-domain (FDTD) 2D simulator for photonic devices (F2P) to find transmission properties on each side of the cavity, ${ }^{16,17}$ and MIT photonicbands package (MPB) to determine dispersion relations and thus emission frequencies. ${ }^{18,19}$ The $n_{\text {eff }}$ method is used to model the lasers in F2P and MPB. Two $n_{\text {eff }}$ are calculated for infinite slab waveguides to represent the minimum and maximum polymer thickness in the structure. This allows threedimensional (3D) structures to be approximated by 2D structures and computational time is reduced significantly. The $n_{\text {eff }}$ method is verified for 3D structures to be approximated by 2D structures in Ref. 20.

A band diagram calculated in MPB for the structure with an imprint depth of $90 \mathrm{~nm}$ is shown in Fig. 2. Lasing is observed at the center frequency of the band gap in the P-direction.

The $\mathrm{PhC}$ lasers are pumped vertically from above with a Q-switched frequency doubled neodynium doped aluminium yttrium garnet laser at $532 \mathrm{~nm}$. The pump laser light is guided to the lasers by an adjustable mirror. This allows the pump light to be angled correctly, thereby coupling to a

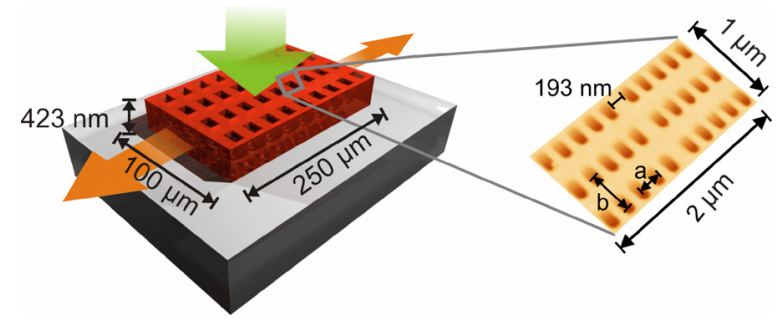

FIG. 1. (Color online) Illustration of an organic dye doped 2D PhC laser. The laser is imprinted with a rectangular lattice $\mathrm{PhC}$ to provide the index contrast, the imprint depth is varied in seven steps in the range [40 nm; 273 $\mathrm{nm}$. The rectangular lattice shown in the polymer is highly enlarged compared to the area of the laser. An AFM picture is included to show the real dimensions of the $\mathrm{PhC}$ and the cavity in the middle of the structure. The arrows represent incoming pump light and emitted laser light. 


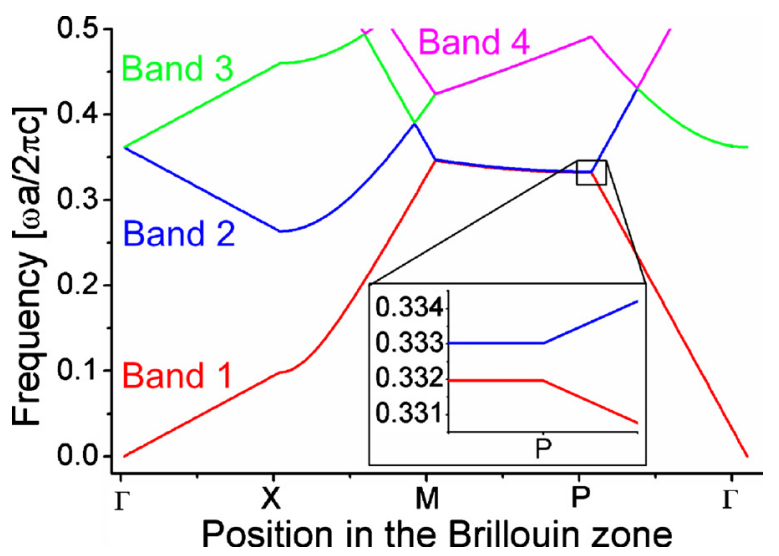

FIG. 2. (Color online) Band diagram calculated in MPB for the structure with $90 \mathrm{~nm}$ imprint depth. Lasing is observed at the frequency corresponding to the middle of the bandgap in the P-direction, see inset.

mode in the plane of the laser. ${ }^{15}$ The laser light is collected by an optical fiber and analyzed with an Ocean Optics HR2000 spectrometer, which has a resolution of $1.5 \AA$. From the spectra the emission wavelength, threshold, and laser lifetime are determined.

The threshold measurements are shown in Fig. 3. The lowest threshold, $3.7 \pm 0.5 \mu \mathrm{J} / \mathrm{mm}^{2}$, is obtained for an imprint depth of $90 \mathrm{~nm}$.

Lifetime is defined in number of pulses as the half-life of the dye molecules and the measurements are shown in Fig. 3. The pump laser intensity is $49 \mu \mathrm{J} / \mathrm{mm}^{2}$, approximately eight times threshold. The longest lifetime $29000 \pm 10000$ pulses is achieved for an imprint depth of 90 $\mathrm{nm}$. Examinations of threshold and lifetime imply that an imprint depth of $90 \mathrm{~nm}$ is optimal. This is investigated further in Fig. 4 where lifetime versus 1/threshold is plotted.

Three representative emission spectra from the lasers are seen in Fig. 5(a), it is evident that not all lasers oscillated in a single-mode. The single-mode yield is plotted as a function of imprint depth and index contrast in Fig. 5(b). The lasers with imprint depths of 40,70, and $90 \mathrm{~nm}$ have a single-mode yield of $93 \%$ and above. Emission spectra have been measured for 23 lasers with an imprint depth of $180 \mathrm{~nm}$, and they all showed two emission peaks resulting in zero single-mode

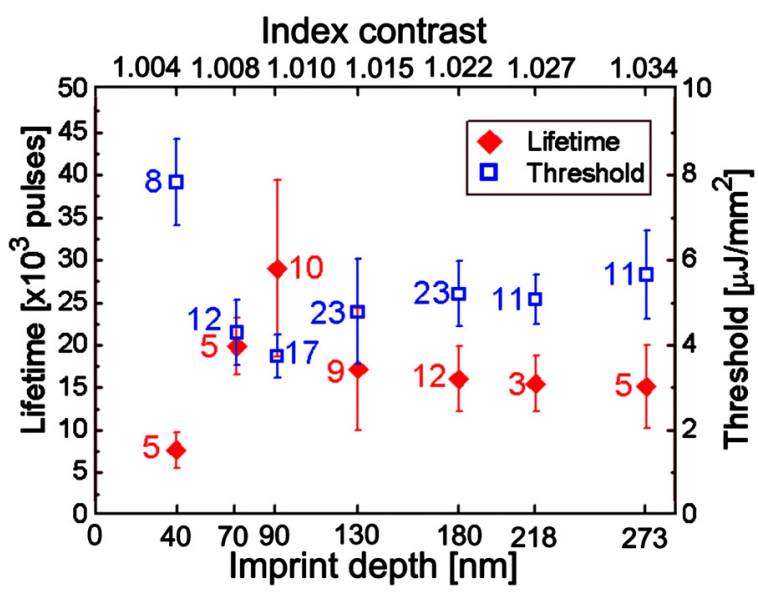

FIG. 3. (Color online) (a) Threshold and lifetime as a function of imprint depth and index contrast. The lowest threshold and longest lifetime is achieved for $90 \mathrm{~nm}$. The pump laser intensity is $49 \mu \mathrm{J} / \mathrm{mm}^{2}$, approximately eight times threshold. The numbers next to the data points indicate the number of examined lasers.

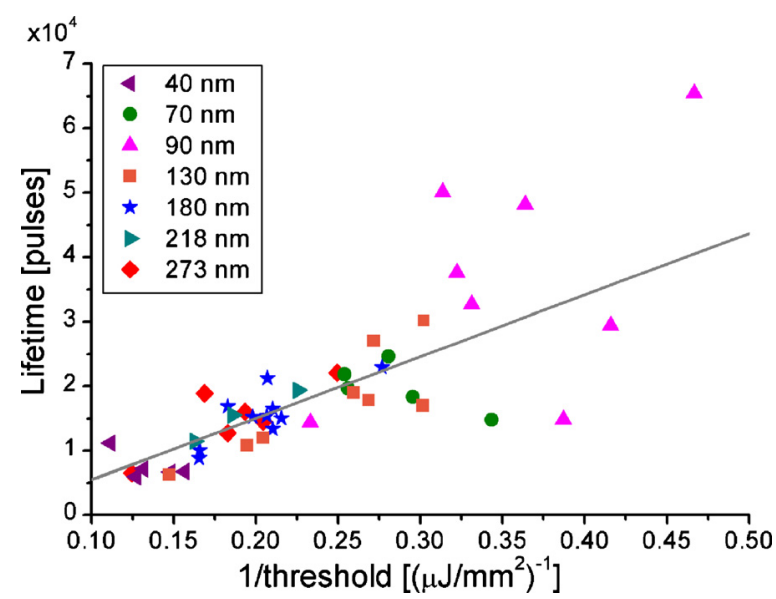

FIG. 4. (Color online) Lifetime as a function of threshold. The inverse proportionality between lifetime and threshold is indicated by a linear fit to the measurement points. Lasers with an imprint depth of $90 \mathrm{~nm}$ have lowest threshold and longest lifetime.

yield. The observed frequencies were spaced with a distance corresponding well to the band gap as seen in Fig. 2. Three peaks were observed for the majority of the lasers with imprint depths of 130, 218, and $273 \mathrm{~nm}$ corresponding to the two band edges and the center frequency of the band gap.

The lowest threshold and the longest lifetime are achieved for an imprint depth of $90 \mathrm{~nm}$. Lower threshold leads to excitation of fewer dye molecules due to gain clamping. Bleaching of the dye occurs when the molecules are excited. ${ }^{21}$ This means that as the population inversion increases the bleaching of the dye will also increase, thereby leading to shorter lifetime of the dye for higher threshold. The proportionality between lifetime and $1 /$ threshold is clarified by a linear fit to the measurement points in Fig. 4. There are some deviations from the fit with the spread mostly pronounced for the imprint depth of $90 \mathrm{~nm}$. The lowest thresholds are observed for this depth resulting in larger spread for 1/threshold. For imprint depths of $130 \mathrm{~nm}$ and above the single-mode yield is less than $40 \%$. We attribute this to the following three possibilities: spatial hole burning, spectral hole burning, or a decrease in the mean free path of the photons. First, spatial hole burning may occur due to localized bleaching and the orthogonality of the modes at the band edges. Second, the possibility of spectral hole burning increases as the imprint depth and band gap increases, which is supported by the larger number of multimode operations seen in the spectra for imprint depths of $130 \mathrm{~nm}$ and above. Third, for an imprint depth of $130 \mathrm{~nm}$, the normalized transmission for one mirror calculated by FDTD at the center frequency is $62 \mathrm{ppm}$, in comparison the normalized transmission is $447 \mathrm{ppm}$ for $90 \mathrm{~nm}$, see Fig. 5(c). The large feedback for imprint depths of $130 \mathrm{~nm}$ could cause small noninteracting domains to be created inside the laser, leading to lasing taking place at more sites independent of each other. When the transmission approaches zero, the mean free path of the photons is reduced significantly preventing them from propagating in the $\mathrm{PhC}$ and possibly interact with the cavity, so the single-mode oscillation is not obtained. Furthermore variations in the polymer height across the device could cause laser action to take place on several modes. The multimode property is one reason for higher threshold and lower lifetime for imprint depths of $130 \mathrm{~nm}$ and above. An index con- 
(a)

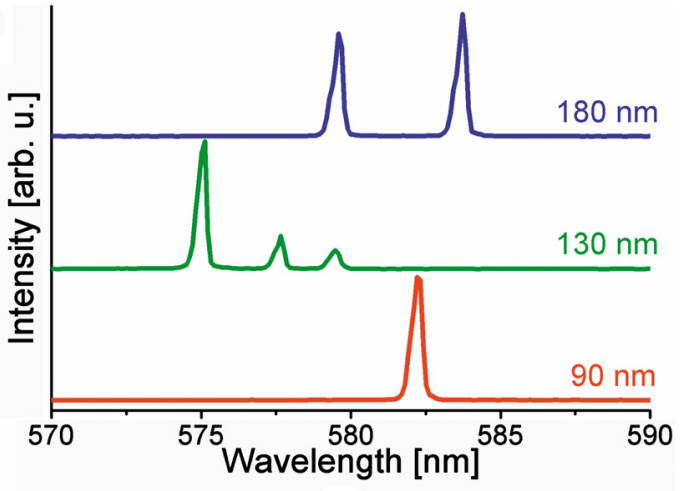

(b)

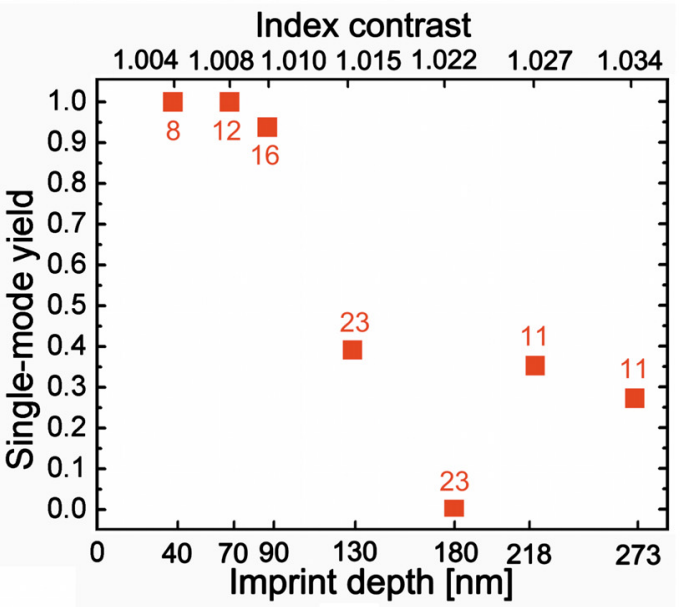

(c)

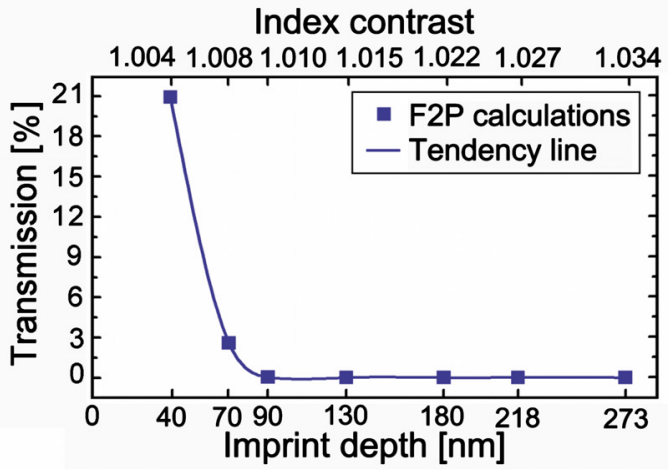

FIG. 5. (Color online) (a) Representative emission spectra for the lasers with imprint depth of 90,130 , and $180 \mathrm{~nm}$. The pump laser intensity is $49 \mu \mathrm{J} / \mathrm{mm}^{2}$ approximately eight times threshold. (b) Single-mode yield as a function of imprint depth and index contrast. For a depth of $90 \mathrm{~nm}$ the yield is $93 \%$, for depths of $130 \mathrm{~nm}$ and above the yield reduces significantly to less than $40 \%$. The numbers indicate the number of examined lasers. (c) Transmission as a function of imprint depth and index contrast calculated with the FDTD method.

trast of 1.010 corresponding to $90 \mathrm{~nm}$ imprint depth gives a single-mode yield of $93 \%$. Therefore this index contrast must be close to the limit of assured single-mode operation. Subsequently, the lasers function optimally with an imprint depth of $90 \mathrm{~nm}$, leading to the lowest threshold and longest lifetime, and $447 \mathrm{ppm}$ seems to be the largest feedback one can attain without losing single-moded operation.

In conclusion, we have investigated Pyrromethene 597 doped Ormocore 2D PhC lasers with seven imprint depths of $\mathrm{PhCs}$ in the range [40 nm; $273 \mathrm{~nm}$ ], corresponding to index contrasts in the range $[1.004 ; 1.034]$. We have identified a trade-off between low threshold and single-mode operation. We have found that the operation threshold increases, lifetime shortens, and the yield of single-mode lasers decreases for imprint depths of $130 \mathrm{~nm}$ and above. The multimode operation can explain the increased threshold and shortened lifetime. We found that an imprint depth of $90 \mathrm{~nm}$ corresponding to an index contrast of 1.010 results in the lowest threshold and longest lifetime, which could be achieved without losing its high single-mode yield of $93 \%$. We have demonstrated that an increase in index contrast does not necessarily lead to a more efficient laser, therefore the imprint depth of the PhC becomes a key parameter in the engineering of 2D PhC lasers.

The partial support from the EC funded project NaPANIL (Project No. NMP-2007-3.5-1, Contract No. 214249) is gratefully acknowledged. M.B.C. is financially supported by the Danish Research Council for Technology and Production Sciences (Grant No. 274-09-0105).

${ }^{1}$ V. Reboud, N. Kehagias, P. Lovera, M. Zelsmann, C. Schuster, F. Reuther, G. Gruetzner, G. Redmond, and C. M. S. Torres, Jpn. J. Appl. Phys. 47, 5139 (2008).

${ }^{2}$ N. Moll, R. F. Mahrt, C. Bauer, H. Giessen, B. Schnabel, E. B. Kley, and U. Scherf, Appl. Phys. Lett. 80, 734 (2002).

${ }^{3}$ S. Balslev and A. Kristensen, Opt. Express 13, 344 (2005).

${ }^{4}$ M. Gersborg-Hansen and A. Kristensen, Appl. Phys. Lett. 89, 103518 (2006).

${ }^{5}$ J. A. Rogers, M. Meier, and A. Dodabalapur, Appl. Phys. Lett. 73, 1766 (1998).

${ }^{6}$ S. Riechel, C. Kallinger, U. Lemmer, J. Feldmann, A. Gombert, V. Wittwer, and U. Scherf, Appl. Phys. Lett. 77, 2310 (2000).

${ }^{7}$ E. Mele, A. Camposeo, R. Stabile, P. Del Carro, F. Di Benedetto, L. Persano, R. Cingolani, and D. Pisignano, Appl. Phys. Lett. 89, 131109 (2006).

${ }^{8}$ P. Del Carro, A. Camposeo, R. Stabile, E. Mele, L. Persano, R. Cingolani, and D. Pisignano, Appl. Phys. Lett. 89, 201105 (2006).

${ }^{9}$ A. Vasdekis, G. Turnbull, I. Samuel, P. Andrew, and W. Barnes, Appl. Phys. Lett. 86, 161102 (2005).

${ }^{10}$ G. Turnbull, P. Andrew, W. Barnes, and I. Samuel, Appl. Phys. Lett. 82, 313 (2003).

${ }^{11}$ H. A. S. Al-shamiri, I. M. Azzouz, M. Salah Shafik, and Y. A. Badr, J. Sol-Gel Sci. Technol. 41, 65 (2007).

${ }^{12}$ E. Yariv, S. Schultheiss, T. Saraidarov, and R. Reisfeld, Opt. Mater. 16, 29 (2001).

${ }^{13}$ Commercially available from microresist technology $\mathrm{GmbH}$, Berlin, Germany, www.microresist.de

${ }^{14}$ X. Cheng and L. J. Guo, Microelectron. Eng. 71, 288 (2004).

${ }^{15}$ M. B. Christiansen, A. Kristensen, S. Xiao, and N. A. Mortensen, Appl. Phys. Lett. 93, 231101 (2008).

${ }^{16}$ M. Qiu, August 2003, www.imit.kth.se/info/FOFU/PC/F2P/index.htm

${ }^{17}$ K. S. Yee, IEEE Trans. Antennas Propag. 14, 302 (1966).

${ }^{18}$ MPB's homepage, March 2009, http://ab-initio.mit.edu

${ }^{19}$ S. G. Johnson and J. D. Joannopoulos, Opt. Express 8, 173 (2001).

${ }^{20}$ M. Qiu, Appl. Phys. Lett. 81, 1163 (2002).

${ }^{21}$ S. Singh, V. R. Kanetkar, G. Sridhar, V. Muthuswamy, and K. Raja, J. Lumin. 101, 285 (2003). 